\title{
Application of Technologies Robotic Rehabilitation in Children with Upper Limb Injury

\author{
Madhu Yadav
}

\begin{abstract}
Robotic and advanced technology rehabilitation is useful for people with difficulties and deficits in arm and hand movements, walking problems and balance disorders. Robotic technologies are being introduced in the rehabilitation field to support the activity of specialists, doctors and physiotherapists; the future and the challenge of rehabilitation lies precisely in the development of robotics. Robot assists the therapist in administering the most appropriate motor therapy with precision and repeatability modulates the difficulty of the exercise. It allows repetitive task-oriented activities with augmentative feedback capable of inducing brain plasticity. It acquires quantitative information on movement and evaluates the services performed he first, "Arm and Hand", is used to help the opening and closing movements of the hand. After entering it by hand and forearm, gently guides the patient's shoulder and elbow movements to reach and grasp objects. "Wrist", on the other hand, interacts with the movements of the wrist and integrates functionally with the "Hand" module.
\end{abstract}

Keywords: Rehabilitations, Robotic therapy, hemiplegia adults, spastic hand, stroke.

\section{INTRODUCTION}

$\mathrm{D}_{\mathrm{i}}$ isability affects quality life span and restrictions on access to education [1]. Is good It is known that children with disabilities generally have difficulties in its Health, low performance education, fewer economic opportunities and greater poverty in adulthood compared to people without disability; as stated by the WHO [2]. Consequently, an economic and social problem can be pointed out called: "Cost of disability" [3].

Robotics is an engineering field that can contribute to solving this problem. This field has been extensively developed and is being applied in a wide variety of settings and fields of human action, such as has been occurring in the rehabilitation field [5]. Fundamentally, to improve of way significant the quality of patients' lives and attend important tasks such as: Surgical interventions of all kinds what includes microsurgery [9], brain accident rehabilitation vascular and cognitive, motor rehabilitation of patients who have suffered limb accidents superiors, robotic assistance for different

\footnotetext{
Manuscript received on 09 April 2021 | Revised Manuscript received on 03 August 2021 | Manuscript Accepted on 15 September 2021 | Manuscript published on 30 September 2021. * Correspondence Author

Dr. Madhu Yadav*, Assistant Professor, IIMT University Meerut (Uttar Pradesh), India. Email: dr.madhu2021@hotmail.com

(C) The Authors. Published by Lattice Science Publication (LSP). This is an open access article under the CC-BY-NC-ND license (http://creativecommons.org/licenses/by-nc-nd/4.0/)
}

tasks, adaptation of intelligent environments for housing for people with disabilities or for adult solder. Currently there are multiple review articles systematic literature in the field of rehabilitation robotics. There are also a large number of systems robotic for upper limb rehabilitation that allow therapy through repetitive tasks in controlled environments, which associated with software specialized playful character allow to maintain high patient motivation. However, there are few developments for the child population and there is still systematically explore and document this field. Because of this, this work is very important because through observational and retrospective synthesis of multiple investigations, will provide information that allow updating new researchers in the field robotic rehabilitation of pediatric patients, and be a starting point for future work. Although an attempt was made to select the most cited, it is very clear that a number of other articles have been omitted due to the extensive bibliography on the subject. Therefore, the aim of this article is to present a review of the status of assistive technologies and its applications for limb rehabilitation superior of children who are in condition of disability

The hand of man, in its complexity, is a structure perfectly logical and adapted to its functions generating countless possibilities of postures, movements and actions that have given him the possibility of perfection due to their mobility, neuromuscular organization and ability to expression

1. From the ancestors it has sought to perfect and facilitate work at an industrial level by means of machines robots to achieve better results in production, up to the point of applying it in the branches of medicine, such as human benefit to solve and facilitate the processes of Recovery. The first robotics concepts appeared in the late 1940s, with the developments of who is considered the father of this discipline G.C. Return who in 1954 he patented the first memory manipulator, giving beginning of the modern era of robotics

2. Regarding the use of robotic therapy as a mechanism functional rehabilitation, the history goes back to the years eighties of the last century, where at first it was not considered that industrial robots were suitable for robotics rehabilitation due to the heaviness of his movements and your accident history; however, in 1999, researchers from Budapest University of Technology and Economics took up the idea of using standard industrial robots for therapy in humans. The REHAROB project was the first in the world looking to use standard industrial robots, manufactured in series, for the physiotherapy of individuals with spastic hemiparesis

ublished By:

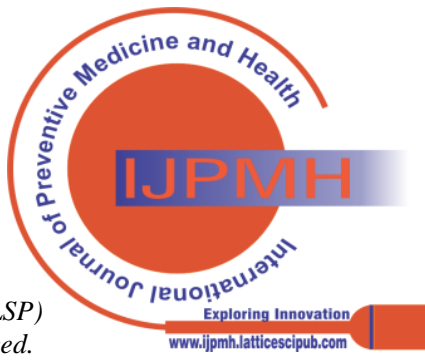


3. Within the development of research on robot-assisted rehabilitation of the upper extremities, found gain in Fugl-Meyer scores (FM) due to changes in functional connectivity in the motor area supplementary, the contralateral motor cortex, ipsilateral, and the parts of the visuospatial system4, positive effects on motor recovery of the upper limb with joint work between robot-assisted therapy and voluntary participation of the motor system of the affected limb5, significant improvements time and speed of movement as a result of greater active ranges of movement and a better index of co-contraction for shoulder extension / flexion 6. Robotic therapy for functional neurorehabilitation of the spastic hand is a term that in recent years It has taken on a lot of strength thanks to advances in technology. The variety of equipment and the competitive costs of themselves have allowed to apply its use as a modality emerging therapy and promises to become a standard within the treatment guidelines. The fact that it is a relatively new technique means that the amount of information available is limited and does not allow to give conclusive results. In addition, the wide variety of equipment makes it difficult to unified analysis of the studies carried out. However, has found that the use of robotic therapy as a complement to conventional therapy and allows obtaining results more effective in people with disease hemiplegia cerebrovascular disease (CVD) [7][8].

\section{MOTOR CONTROL PRINCIPLES AND ROBOTIC} THERAPY

The training of these devices is based on different exercise modalities that can help restore motor control of the upper limb and its function. One of the modalities is passive movement, in which the robotic device moves the person's arm (possible in all robotic devices), on the other hand is the movement active or partially assisted or resisted by the device robotic for the case of voluntary and selective function (in the MIT-Manus, Bi-Manu-Track, MIME) [8]. One modality additional is the bimanual exercise, in which the movement active arm of the unaffected arm is reflected in passive movement simultaneous of the affected arm (in Bi-Manu-Track and MIME). In most robotic systems, more than a modality in a single device, most were designed for training the proximal part of the limb superior by allowing movement in multiple directions, meanwhile devices such as the Bi-Manu-Track focuses in the distal part of the upper limb and the MIT-Manus that was designed for training the movements of the doll. New robotic devices and evolutions of existing ones are continually being developed, for example, Furusho and Colombo who include various systems hand movement training11

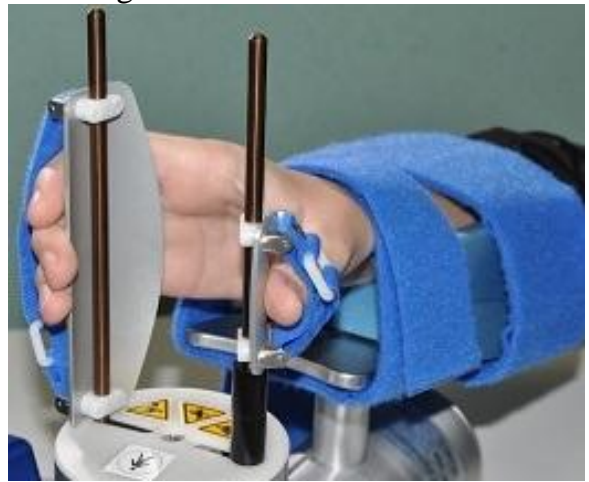

Fig.1. Child Neurorehabilitation Robot

\section{Motor Learning, Neuroplasticity and Robotic Therapy}

The systematic application of robotics in neuro rehabilitation comes directly from recent discoveries of the neuroscience that clearly demonstrate how exercises physical movements based on voluntary movements are capable of produce significant clinical outcomes in recovery after suffering a CVD. In fact, these exercises not only promote functional recovery after a traumatic injury to the Central Nervous System (CNS) 12, but they also promote the neurogenesis process13. For other part, active exercises stimulate mechanisms, mediate by brain neurotrophic factors, which improve neural plasticity through the use of robotic devices, such as possible rehabilitation strategy to achieve recovery motor, is justified in its impact on improving treatment therapeutic and in the learning of motor function. In any case, the therapeutic approach must be well structured and be repetitive, in order to promote cortical reorganization after ECV14. Recommendations of the American Heart Association (AHA) on comprehensive Rehabilitation and multidisciplinary of people who have suffered a stroke, provides an overview and the best available evidence for different medical treatments after CVD, including traditional rehabilitation therapies and newer techniques, such as robot-assisted therapies15,16.

Currently in Colombia, robotic systems are having perfected in an exponential way, both in the development of different job applications, assistance, environmental surveys, among others; as well as of progress in artificial intelligence methods applied and specialized hardware for specific tasks that previously did not could be developed.

There is growing interest in the use of robotic devices in neurorehabilitation, for example, at the level of upper limbs has been found to function of the paretic arm in activities of daily living can improve in people with CVD.

The principles of control and motor learning of form computational data derived from studies in healthy subjects introduced in the context of robotic neurorehabilitation. Special attention is paid to the idea of context, the generalization of tasks and training schedule, are examined the assumptions underlying the choice of trajectories of programmed movement in therapy training robotics and the degree of active participation required. We view rehabilitation as a learning disability in general, and is examined from the perspective of frameworks theoretical as supervised and unsupervised learning. Robotic devices today are helping to show great results on people with injuries to level of motor control and these technologies have opened new fields of action in the treatment of the upper limb. The Research is gaining ground in the interpretation of nervous system and how it builds internal models of neuroplasticity. Low impedance and control strategies through feedback, robots are also being used to help in the practice of a repetitive movement providing information about it and allowing recovery after injury, a role played the robotic system in the brain, especially in the cortex somatosensory and motor control servomechanisms.

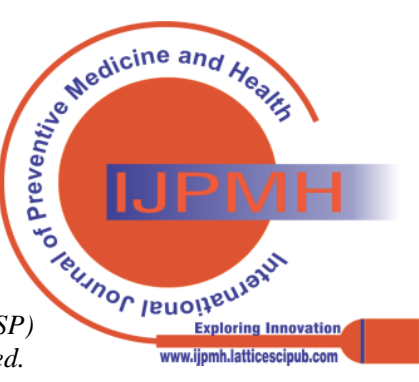


These devices may have the ability to assess also sensorimotor performance as well as quantify the quantity and quality of training improving control motor with a higher level of recovery, generating paradigms that can eventually improve learning where rehabilitation goes beyond the possible levels that if performed with conventional training techniques18.

\section{THERAPY COMPONENTS ROBOTICS}

The use of robots in rehabilitation has two applications fundamental: during active movements of the patient, allow to store information on the performance of the movement (position, speed and force), helping this way to evaluate the evolution of therapy on people19. The final objective of the self-adaptive robotic rehabilitation system is the incorporation of information from user and system control. For this it will be done use of information extracted from physiological signals of the person to estimate their psychophysiological state and use this information to automatically modify the level of Difficulty of the task 20. As robotic components in the rehabilitation of developed so far are: HEXXOR, which consists of two modules that allow separate control finger and thumb movements, accommodates different hand sizes and provides strength assistance from according to the patient's needs, there are also glove-type devices, such as the GLOREHA that leaves the palm is completely free, has little weight and has a software that allows 3D representation of the movements made. Other devices like the Hand of Hope $(\mathrm{HOH})$ allow self-initiation of movement through electromyographic signals (EMG) and offers simulation of different daily activities21. Finally, the AMADEO provides different levels of flex and extension strengths of each finger, while measuring ranges of motion22.

\section{PROCEDURES FUNCTIONAL NEUROREHABILITATION WITH ROBOTIC THERAPY}

The most important advantage of using robotic technology in functional neurorehabilitation interventions, it is the high dose and high intensity training delivery capacity, therefore rehabilitation robots are divided in therapeutic and assistive robots, whose purpose is the compensation, while therapeutic robots provide task-specific training7, according to Edmans "Robotic systems must use reality systems mixed, where motion and vision sensitive objects artificially create a virtual reality environment that is driven by the manipulation of real objects. Robots' rehabilitation can be divided into therapeutic robots, which provide task-specific training and assistance robots whose purpose is compensation, these devices have the potential for greater impact on the decrease in impairment with characteristics such as applicability across a wide range of motor impairments, high reliability in measurement, management of therapeutic doses, training protocols and easy implementation. Automation and robotics are making it possible to perform tasks in assisted mode with the help of devices advanced assistants with special emphasis on support to biomechanical functions where bioengineering constitutes a multidisciplinary area of work and research and therefore offers important knowledge and tools to address issues related to diagnosis and the follow-up of the different pathologies with needs functionalities and the diversity of therapies to be applied, therefore the robotics for rehabilitation handles different systems for the improvement of clinical therapy in patients with CVD, claiming to improve muscle tone, joint mobility and neuromotor coordination, preventing impairments such as atrophy and osteoporosis. Most of these types of robots they are a type of orthosis that tries to replicate a movement through passive or active mobility, managing to assist the patient in his or her deficient movements or supporting him, as for example the hand built by Dicicco with EMG control and the Sasaki orthosis, in all cases the possibilities of rehabilitation that opens the robotics are very broad because it can only help the user to perform the functions biomechanics in the right measure but the therapist can establish a specific evolutionary program for each patient and also assess the progress achieved in each session by measuring representative biomechanical parameters such as linear amplitude of motion or angular, execution times, forces and torques exerted24. The biomechanical prediction block allows anticipation of the errors that the patient may commit. East subsystem is based on the so-called "forward models" present in the cerebellum, which transform commands motor in sensory consequences to estimate the state of the body and its environment. These models are necessary, mainly, because human transmission lines (axons) carry information at a slower speed to that of the speed of sound, so it must be compensated the accumulated delay in perceived sensory information. Robotic neurorehabilitation is therefore attractive because to various reasons such as its great potential for easy deployment, its applicability in a wide range of deficits engines and their reliability in taking measurements, which has led to a large increase in the penetration of these devices in clinical settings. In addition, it is also believing that robot-based therapies during the acute phase and subacute favors functional recovery due to spontaneous biological processes [25].

\section{ACTUAL TRENDS IN ROBOTIC THERAPY}

The most recent research shows that the combination of a conventional movement treatment passive combined with one assisted by a robotic device generates significant gains not only in the improvement of my articular conditions of the individual but also in their functionality giving clarity that despite research made, each one separately does not obtain greater results As when combined, these devices must be safe, not very complex and light, to allow their proper use26. In hospital phases it is already venturing into the treatment with robotic equipment in order to avoid a establishment of the spastic phase in people, generating a better future forecast for the mobility of the segment. It is currently showing how research in this broad topic has grown rapidly and the number of robots therapeutic rehabilitation has expanded dramatically in the last two decades26. Likewise, it is important to note that no robotic device replaces the action of the health professional, on the contrary becomes a more supportive element for the rehabilitation of people, giving the professional an adequate dosage of the sessions in terms of speed, endurance and intensity of it measures the evolution according to the time range that is desired.

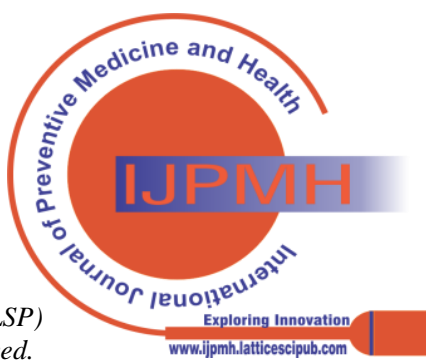




\section{MATERIAL AND METHODS}

As part of the input for the preparation of a review systematic about the use of robotic therapy for the treatment of adult spastic hand with hemiplegia, the construction of the theoretical reference of the investigation, for which a search process was carried out of scientific literature in PubMed, Cochrane Central Register of Controlled Clinical Trials CENTRAL, LILACS, Scielo, as well as, in virtual libraries, ScienceDirect, ProQuest, SpringerLink, BioMed Central, Occupational Therapy Systematic Evaluation of Evidence (OTSEEKER), Physiotherapy Evidence Database, such searches were carried out in the period between July 24 and on December 16, 2020; the inclusion criteria were: randomized controlled trials conducted in adults with Spastic hemiplegia due to CVD between 18 and 75 years of age and robotic therapy as a neurorehabilitation technique used for upper limb training.

\section{RESULT}

Clinical description. A 20-year-old female patient, a psychology student and a worker in a telephone call center, accidentally hit a wall with his right hand, suffered a complex intra-articular fracture of the head of the first metacarpal, 5 days after the trauma she underwent open reduction plus osteosynthesis and 7 days after surgery he presented total functional limitation of the thumb and partial functional limitation of the second finger, caused by pain and edema Intervention.

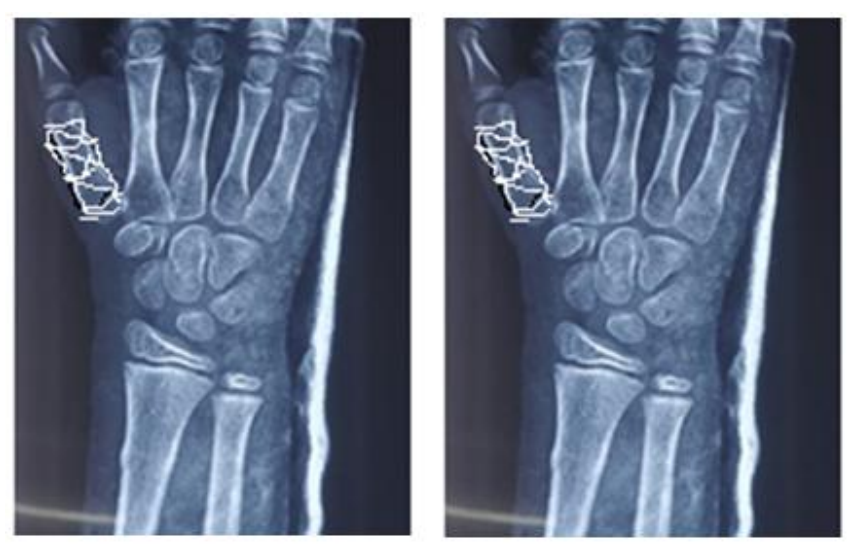

Fig. 2. Pain and Edema

After 10 days after surgery, on 22/12/2020, the rehabilitation process began; 10 sessions of physical therapy lasting 60 minutes each and 12 sessions of mobilization therapy assisted with robotic orthoses each lasting 60 minutes.

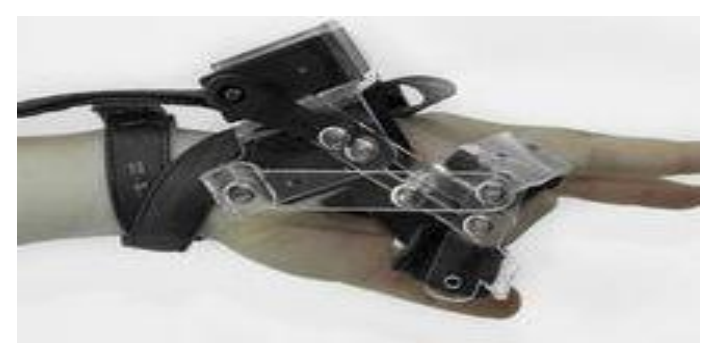

Amount of the Disabilities of the Arm, Shoulder and Hand and Visual Analog Scales in the first patient who presented a trauma at home on 22/12/2020. She

\begin{tabular}{|c|c|c|}
\hline Variables & Function & Pain \\
\hline $\begin{array}{c}\text { Measurement I on } \\
\text { 22/12/2020 }\end{array}$ & 69.85 & 3 \\
\hline $\begin{array}{c}\text { Measurement II on } \\
\text { 15/01/2021 }\end{array}$ & 14.7 & 2 \\
\hline
\end{tabular}

Number of arches of joint motion in the first patient

\section{DISCUSSION}

It is recognized that hemiplegia generally occurs as consequence of CVD, defined according to the Organization World Health as' the rapid development of signs focal (or global) impairment of brain function, with symptoms lasting 24 hours or longer, or leading to death without other causes the vascular origin27 "whose motor deficiency is characterized by compromise of the contralateral hemibody to the site of injury, which may be accompanied by alterations sensitive, cognitive, perceptual and language. In hemiplegia it is common to find involvement of the function of the upper limbs leading not only to difficulty in performance of selective movement patterns, but by functional component, individual and bilateral, especially for the manual function and although the potential of recovery there are intervention methods used that may decrease levels of disability and dependency as a result of limb dysfunction superiors. In this sense, much of the knowledge.

\section{CONCLUSIONS}

This review article presented the advances who has conceived rehabilitation engineering through of robotic rehabilitation for children with injury to the superior member. This was considered when studying the reported literature, where each year they develop new therapy devices, software and protocols; although many of these developments are scarce to children or do not have any application in the population childish. However, in different studies reported in the specialized literature, therapies with systems robotics are proving to be just as effective compared to conventional therapies, under the same intensity parameters and treatment time. No However, it should be understood that the purpose of robots in therapies is to be a tool, at no time can replace the role of the physiotherapist or doctor specialist to guide therapy and interpret results. Without denying the importance of autonomy in robotic systems and their playful nature, which allow maintain a high motivation on the part of the patient Robotic device-mediated physiotherapy offers a new possibility to improve the result of rehabilitation of people recovering from CVD, the device-supported upper limb training robotic devices involving the shoulder, elbow and wrist show Significant improvement in the reduction of spasticity measured according to the modified Ashworth scale and with it a subsequent improvement in motor control for the execution of different activities. In recent years there has been a dizzying advance in the use of robotic therapy for hand rehabilitation generating next-generation devices that enter play a very important role in the recovery process of patients with different types of impairment in hand functionality due to brain injury.

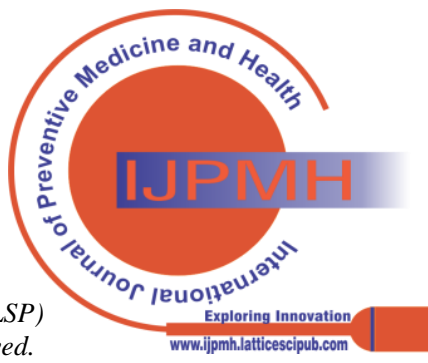


These alterations that generate increased muscle tone and in a hypertonia that leads to generate a spastic picture limiting the individual for the execution of functional patterns not only of the hand but of the entire upper limb. On the other hand, it was observed that robotic rehabilitation has its own approach to technical problems faced by engineers and designers; this means than to develop new rehabilitation systems robotics, it is required to take into account the following: adaptability to the end user, energy consumption and the acceptability of the technology by therapists and specialized rehabilitation institutions. Finally, although technological advances in robotic rehabilitation for children are on the rise, currently it is necessary greater availability of systematic information, increase of the component interdisciplinary, problem delimitation, feedback of results, recreational therapies; as well as, establish alliances with specialized institutions of Health.

\section{REFERENCES}

1. MaciejaszP, EschweilerJ, Gerlach-HahnK, et al. A survey on robotic devices for upper limb rehabilitation. J Neuroeng Rehabil. 2014;11(1):3-29. [Crossref], [PubMed], [Web of Science ${ }^{\circledR}$ ], [Google Scholar]

2. GopuraRARC, BandaraDSV, KiguchiK, et al. Developments in hardware systems of active upper-limb exoskeleton robots: a review. Rob Auton Syst. 2015;75:203-220. [Crossref], [Web of Science ${ }^{\circledR}$ ], [Google Scholar]

3. MolteniF, GasperiniG, CannavielloG, et al. Exoskeleton and end-effector robots for upper and lower limbs rehabilitation: narrative review. PM R. 2018;10(9):S174-S188. [Crossref], [PubMed], [Google Scholar]

4. GrimaldiG, MantoM. Functional impacts of exoskeleton-based rehabilitation in chronic stroke: multi-joint versus single-joint robotic training. J Neuroeng Rehabil. 2013;10(1):2-4. [Crossref], [PubMed], [Google Scholar]

5. FeiginVL, RothGA, NaghaviM, et al. Global burden of stroke and risk factors in 188 countries, during 1990-2013: a systematic analysis for the global burden of disease study 2013. Lancet Neurol. 2016;15(9):913-924. [Crossref], [PubMed], [Web of Science ${ }^{\circledR}$ ], [Google Scholar]

6. “About Stroke," 2019. [Online]. [cited: 27 Nov 2019]. Available from: https://www.stroke.org/en/about-stroke. [Google Scholar]

7. MannaSK, BhaumikS. A bioinspired 10 DOF wearable powered arm exoskeleton for rehabilitation. J Robot. 2013;2013:1-15. [Crossref], [Google Scholar]

8. LoK, StephensonM, LockwoodC. Mobility and functional ability in adult stroke patients: a systematic review protocol. JBI Database System Rev Implement Rep. 2017;15(12):3049-3091. [Crossref], [PubMed], [Google Scholar]

9. DuretC, GrosmaireA, KrebsHI. Robot-assisted therapy in upper extremity hemiparesis: overview of an evidence-based approach. Front Neurol. 2019;10(April):1-8. [Crossref], [PubMed], [Google Scholar]

10. LoseyDP, McDonaldCG, BattagliaE, et al. A review of intent detection, arbitration, and communication aspects of shared control for physical human-robot interaction. Appl Mech Rev. 2018;70(1):10804. [Crossref], [Web of Science ®], [Google Scholar]

11. BirouaFI. Development and testing of a mixed feedback control system for robotic hand exoskeleton. 2019 15th international conference on engineering of modern electric systems (EMES); IEEE; 2019. p. 17-20 [Crossref], [Google Scholar]

12. PirondiniE, CosciaM, MarcheschiS, et al. Evaluation of the effects of the arm light exoskeleton on movement execution and muscle activities: a pilot study on healthy subjects. J Neuroeng Rehabil. 2016;13(1):1-21. [Crossref], [PubMed], [Google Scholar]

13. KyleD. Clinical evaluation of an upper limb exoskeleton for rehabilitation after incomplete spinal cord injury [PhD diss]. Rice University, 2015. [Google Scholar]

14. BertaniR, MelegariC, De ColaMC, et al. Effects of robot-assisted upper limb rehabilitation in stroke patients: a systematic review with meta-analysis. Neurol Sci. 2017;38(9):1561-1569. [Crossref], [PubMed], [Web of Science ${ }^{\circledR}$ ], [Google Scholar]
15. SinghH, UngerJ, ZariffaJ, et al. Robot-assisted upper extremity rehabilitation for cervical spinal cord injuries: a systematic scoping review. Disability Rehabil Assistive Technol. 2018;13(7):704-715. [Crossref], [Taylor \& Francis Online], [Web of Science ${ }^{\circledR}$ ], [Google Scholar]

16. XiaoF, GaoY, WangY, et al. Design and evaluation of a 7-DOF cable-driven upper limb exoskeleton. J Mech Sci Technol. 2018;32(2):855-864. [Crossref], [Web of Science ${ }^{\circledR}$ ], [Google Scholar]

17. HuangJ, TuX, HeJ. Design and evaluation of the RUPERT wearable upper extremity exoskeleton robot for clinical and in-home therapies. IEEE Trans Syst Man, Cybern: Syst. 2016;46(7):926-935. [Crossref], [Web of Science ${ }^{\circledR}$ ], [Google Scholar]

18. WuQ, WangX, ChenB, et al. Patient-active control of a powered exoskeleton targeting upper limb rehabilitation training. Front Neurol. 2018;9(October):1-13. [Crossref], [PubMed], [Google Scholar]

19. FrisoliA, SotgiuE, ProcopioC, et al. Training and assessment of upper limb motor function with a robotic exoskeleton after stroke. 2012 4th IEEE RAS \& EMBS international conference on biomedical robotics and biomechatronics (BioRob); 2012. p. 1782-1787. [Crossref], [Google Scholar]

20. PéterO, FazekasG, ZsigaK, et al. Robot-mediated upper limb physiotherapy: review and recommendations for future clinical trials. Int J Rehabil Res. 2011;34(3):196-202. [Crossref], [PubMed], [Web of Science ${ }^{\circledR}$ ], [Google Scholar]

21. MehrholzJ, HädrichA, PlatzT, et al. Electromechanical and robot-assisted arm training for improving activities of daily living, arm function, and arm muscle strength after stroke. Cochrane Database Syst Rev. 2015 (11). [Crossref], [Web of Science ${ }^{\circledR}$ ], [Google Scholar]

22. ShengB, ZhangY, MengW, et al. Bilateral robots for upper-limb stroke rehabilitation: state of the art and future prospects. Med Eng Phys. 2016;38(7):587-606. [Crossref], [PubMed], [Web of Science (®), [Google Scholar]

23. VeerbeekJM, Langbroek-AmersfoortAC, Van WegenEEH, et al Effects of robot-assisted therapy for the upper limb after stroke. Neurorehabil Neural Repair. 2017;31(2):107-121. [Crossref], [PubMed], [Web of Science ${ }^{\circledR}$ ], [Google Scholar]

24. ZhangC, Li-TsangCWP, AuRKC. Robotic approaches for the rehabilitation of upper limb recovery after stroke: a systematic review and meta-analysis. Int J Rehabil Res. 2017;40(1):19-28. [Crossref], [PubMed], [Web of Science ${ }^{\circledR}$ ], [Google Scholar]

25. XiloyannisM, ChiaradiaD, FrisoliA, et al. Physiological and kinematic effects of a soft exosuit on arm movements. J Neuroeng Rehabil. 2019;16(1):1-15. [Crossref], [PubMed], [Google Scholar]

26. GassertR, DietzV. Rehabilitation robots for the treatment of sensorimotor deficits: a neurophysiological perspective. J Neuroeng Rehabil. 2018;15(1):1-15. [Crossref], [PubMed], [Google Scholar]

27. LoAC, GuarinoPD, RichardsLG, et al. Robot-assisted therapy for long-term upper-limb impairment after stroke. $\mathrm{N}$ Engl J Med. 2010;362(19):1772-1783. [Crossref], [PubMed], [Web of Science (]), [Google Scholar]

28. RienerR, LünenburgerL, ColomboG. Human-centered robotics applied to gait training and assessment. J Rehabil Res Dev. 2006;43(5):679-693. [Crossref], [PubMed], [Google Scholar]

29. DietzV, FouadK. Restoration of sensorimotor functions after spinal cord injury. Brain. 2014;137(3):654-667. [Crossref], [PubMed] [Google Scholar]

30. Marchal-CrespoL, McHughenS, CramerSC, et al. The effect of haptic guidance, aging, and initial skill level on motor learning of a steering task. Exp Brain Res. 2010;201(2):209-220. [Crossref], [PubMed], [Web of Science ${ }^{\circledR}$ ], [Google Scholar]

\section{AUTHORS PROFILE}

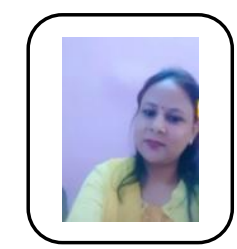

Dr. Madhu Yadav, is working as an Assistant Professor in Physiotherapy Department IIMT University Meerut UP India since 2019. 